\title{
Analysis of Fractional Difference Schemes with Application to Radiographic Images
}

\author{
Milorad P. Paskaš, Member, IEEE, Branimir D. Reljin, Senior Member, IEEE, and Irini S. Reljin, \\ Senior Member, IEEE
}

\begin{abstract}
Visual inspection of radiographic images by radiologists is a regular practice in making a diagnosis. Thus the enhancement of details in radiographs can improve inspection and diagnosis certainty. Through this paper we perform the analysis of the fractional gradient for visual improvement of chest radiographs. Two implementations of the fractional derivative operator, based on central fractional differences, are evaluated. Also we tested two norms for calculation of the magnitude of the fractional gradient, Euclidean and infimum norm, and the conducted tests for both norms are consistent.
\end{abstract}

Keywords - Fractional calculus, fractional derivative, gradient operators, image enhancement, radiographic images.

\section{INTRODUCTION}

$F^{n}$ RACTIONAL derivative generalizes the notion of integerorder derivatives in case of non-integer (fractional) orders of differentiation [1]. Various orders of differentiation, $\alpha$, make a twofold impact on images [2], [3]: integration behavior is present for orders $-1<\alpha<0$ , and detail enhancement in case $0<\alpha<1$. Existing generalizations of the fractional derivative operator are restricted by different conditions [4]. There are various definitions of fractional derivatives in literature [5]. In this paper we use the Grünwald-Letnikov operator based on a central fractional difference from [6].

The scope of the paper is to evaluate two possible

Paper received April 13, 2018; revised June 18, accepted June 20, 2018. Date of publication July 31, 2018. The associate editor coordinating the review of this manuscript and approving it for publication was Prof. Ljiljana Milić.

This paper is a revised and expanded version of the paper presented at the 25rd Telecommunications Forum TELFOR 2017 [11].

The research presented in this paper is partially funded by Ministry of Education, Science and Technological Development of the Republic of Serbia as a project TR32048, and Bilateral Project with Republic of Belarus on high dynamic image compression.

Research presented in this paper was partially supported by the Ministry of Education, Science and Technological Development, Government of the Republic of Serbia under Project III 44009.

Corresponding author Milorad P. Paskaš, Innovation Center, School of Electrical Engineering, University of Belgrade, Bulevar kralja Aleksandra 73, 11120 Belgrade, Serbia (e-mail: milorad.paskas@ic.etf.rs).

Branimir D. Reljin, School of Electrical Engineering, University of Belgrade, Bulevar kralja Aleksandra 73, 11120 Belgrade, Serbia; (e-mail: reljinb@etf.rs).

Irini S. Reljin, School of Electrical Engineering, University of Belgrade, Bulevar kralja Aleksandra 73, 11120 Belgrade, Serbia; (e-mail: irini@etf.rs) generalizations of 1D central fractional difference scheme for 2D signals: 1) direct implementation from [6], and 2) generalization proposed in this paper inspired by the popular Sobel operator [7]. We further perform experiments on chest radiographs and analyze parameters of the model for enhancement of radiographic images for better visual investigation.

The paper is organized as follows. Section II introduces central derivative operator of the fractional order through central fractional differences. It is given in 1D form, while Section III brings two possible realizations of the fractional operator. The first one is the straightforward implementation of the 1D central differences and the other implementation is the generalization of the Sobel operator widely used as a first-order gradient. Evaluation of 2D masks is evaluated on radiographic images in Section IV and the numerical results are discussed. We implement the calculation of gradient using two norms - Euclidean and infimum norm. Finally, Section $\mathrm{V}$ gives conclusions and potential directions in further research on this topic.

\section{II.CENTRAL FraCtional DIFFERENCE}

Central difference, $\delta_{C}$, defined on integer mesh points, in case of integer orders of differentiation, is calculated as [6]:

$$
\delta_{C}^{a}=\left(E_{h}-E_{h}^{-1}\right)^{a}
$$

where $a \in \mathbb{N}$ is the order of differentiation and $E$ is a translation or shifting operator defined as:

$$
E_{\mu h} f(x)=f(x+\mu h)
$$

where $h$ is an elementary shift, $\mu$ is a scalar, $x \in \mathbb{R}$ and $f: \mathbb{R} \rightarrow \mathbb{R}$. Binomial expansion of (1) leads further to:

$$
\delta_{C}^{a}=\sum_{k_{0}=0,1,2, \ldots}^{a}\left(\begin{array}{l}
a \\
k_{0}
\end{array}\right)(-1)^{k_{0}} E^{a-2 k_{0}}
$$

Substituting $k=a-2 k_{0}$ into (3) gives:

$$
\delta_{C}^{a}=\sum_{k=-a,-a+2 \ldots}^{a}\left(\frac{a-k}{2}\right)(-1)^{\frac{a-k}{2}} E^{k},
$$

or:

$$
\delta_{c}^{a}=\sum_{k=-a,-a+1 \ldots}^{a}\left(\frac{a-k}{2}\right) \operatorname{sgn}\left\{\cos \left(\frac{a-k}{2} \pi\right)\right\} E^{k} .
$$

Finally, fractional derivative of order $\alpha \in \mathbb{R}, \alpha>-1$ defined with central differences on integer mesh points is calculated as: 


$$
D_{C}^{\alpha} f(x)=\lim _{h \rightarrow 0} \frac{1}{|2 h|^{\alpha}} \sum_{k=-\infty}^{\infty} g_{k} f(x+k h)
$$

where the coefficients within the summation sign are:

$$
g_{k}=\operatorname{sgn}\left\{\cos \left(\frac{\alpha-k}{2} \pi\right)\right\}\left(\begin{array}{c}
\alpha \\
\left.\frac{\alpha-k}{2}\right)
\end{array} .\right.
$$

The Fourier transform of the differential operator from (6) is [6]:

$$
F\left\{D_{C}^{\alpha}\right\}=\left\{\begin{array}{l}
(j \omega)^{\alpha}, \cos \frac{\alpha \pi}{2}=0 \\
\frac{(j \omega)^{\alpha}}{\left|\cos \frac{\alpha \pi}{2}\right|}, \text { otherwise. }
\end{array}\right.
$$

This shows that central difference scheme from (7) preserves transfer function of an ideal differentiator up to a scale factor defined by the order of differentiation.

\section{IMAGE FRACTIONAL GRADIENT MASKS}

In this paper we assume the derivative masks of fractional order only of the odd size, that is, of the size $(2 N+1) \times(2 N+1)$. In this way the central pixel within the mask is in its center and the symmetry of the neighborhood pixels is provided. Masks of the even size can be obtained in a similar way, while the symmetry with respect to the central pixel cannot be preserved.

\begin{tabular}{|c|c|c|c|c|}
\hline 0 & 0 & 0 & 0 & 0 \\
\hline 0 & 0 & 0 & 0 & 0 \\
\hline$g_{-2}$ & $g_{-1}$ & $g_{0}$ & $g_{1}$ & $g_{2}$ \\
\hline 0 & 0 & 0 & 0 & 0 \\
\hline 0 & 0 & 0 & 0 & 0 \\
\hline
\end{tabular}

\begin{tabular}{|c|c|c|c|c|}
\hline 0 & 0 & $g_{-2}$ & 0 & 0 \\
\hline 0 & 0 & $g_{-1}$ & 0 & 0 \\
\hline 0 & 0 & $g_{0}$ & 0 & 0 \\
\hline 0 & 0 & $g_{1}$ & 0 & 0 \\
\hline 0 & 0 & $g_{2}$ & 0 & 0 \\
\hline
\end{tabular}

Fig. 1. Fractional derivative masks of the size $5 \times 5$ pixels ( $N=2$ ) obtained by direct implementation of the central difference schemes on images.

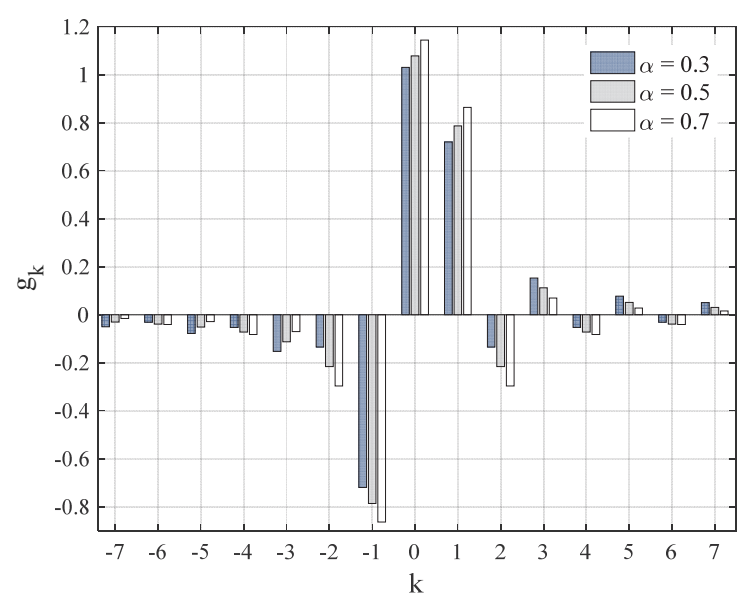

Fig. 2. Coefficients of the direct implementation mask $\left(g_{k}\right)$ calculated for three values of fractional order of differentiation: $\alpha=0.3 ; 0.5 ; 0.7$.

\section{A. Direct Implementation Masks}

Fractional derivative (gradient) masks are defined for $x$ and $y$ spatial directions, respectively, as:

$$
\mathbf{G}_{\mathbf{x}}=\left[\begin{array}{llllllll}
0 & \cdots & 0 & 1 & 0 & \cdots & 0
\end{array}\right]_{(2 N+1) \times 1}^{\prime} \cdot\left[g_{-N} \cdots g_{0} \cdots g_{N}\right]
$$

and

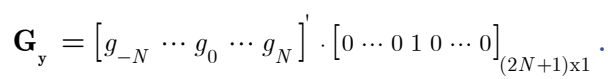

Equations (9) and (10) are derived directly from (6) and these masks are illustrated in Fig. 1. Depicted masks show that image, i.e. 2D signal, is treated as a $1 \mathrm{D}$ signal per each spatial direction, without involving diagonal pixels from the neighborhood (zeros in the masks). The coefficients are numerically presented in Fig. 2 for three orders of differentiation. It is evident that coefficients decrease rapidly as moving from the central pixel $(k=0)$.

\section{B. Masks Implementation with Weighting}

Here we propose involving diagonal pixels within the derivative mask. This is done by analogy with the Sobel operator where the central row (for $x$-direction) or column ( $y$-direction) is copied over the entire mask and then weighted with the weights matrix, $\mathrm{W}_{\mathrm{N}}$ :

$$
\begin{aligned}
& \mathbf{G}_{\mathbf{x}}^{m}=\mathbf{W}_{\mathbf{N}}^{\prime} \cdot\left[\begin{array}{lllll}
g_{-N} & \cdots & g_{0} & \cdots & g_{N}
\end{array}\right] \\
& \mathbf{G}_{\mathbf{y}}^{m}=\left[g_{-N} \cdots g_{0} \cdots g_{N}\right]^{\prime} \cdot \mathbf{W}_{\mathbf{N}} .
\end{aligned}
$$

\begin{tabular}{|l|l|l|l|l|}
\hline $\mathrm{w}_{-2} g_{-2}$ & $\mathrm{w}_{-2} g_{-1}$ & $\mathrm{w}_{-2} g_{0}$ & $\mathrm{w}_{-2} g_{1}$ & $\mathrm{w}_{-2} g_{2}$ \\
\hline $\mathrm{w}_{-1} g_{-2}$ & $\mathrm{w}_{-1} g_{-1}$ & $\mathrm{w}_{-1} g_{0}$ & $\mathrm{w}_{-1} g_{1}$ & $\mathrm{w}_{-1} g_{2}$ \\
\hline $\mathrm{w}_{0} g_{-2}$ & $\mathrm{w}_{0} g_{-1}$ & $\mathrm{w}_{0} g_{0}$ & $\mathrm{w}_{0} g_{1}$ & $\mathrm{w}_{0} g_{2}$ \\
\hline $\mathrm{w}_{1} g_{-2}$ & $\mathrm{w}_{1} g_{-1}$ & $\mathrm{w}_{1} g_{0}$ & $\mathrm{w}_{1} g_{1}$ & $\mathrm{w}_{1} g_{2}$ \\
\hline $\mathrm{w}_{2} g_{-2}$ & $\mathrm{w}_{2} g_{-1}$ & $\mathrm{w}_{2} g_{0}$ & $\mathrm{w}_{2} g_{1}$ & $\mathrm{w}_{2} g_{2}$ \\
\hline
\end{tabular}

\begin{tabular}{|l|l|l|l|l|}
\hline $\mathrm{w}_{-2} g_{-2}$ & $\mathrm{w}_{-1} g_{-2}$ & $\mathrm{w}_{0} g_{-2}$ & $\mathrm{w}_{1} g_{-2}$ & $\mathrm{w}_{2} g_{-2}$ \\
\hline $\mathrm{w}_{-2} g_{-1}$ & $\mathrm{w}_{-1} g_{-1}$ & $\mathrm{w}_{0} g_{-1}$ & $\mathrm{w}_{1} g_{-1}$ & $\mathrm{w}_{2} g_{-1}$ \\
\hline $\mathrm{w}_{-2} g_{0}$ & $\mathrm{w}_{-1} g_{0}$ & $\mathrm{w}_{0} g_{0}$ & $\mathrm{w}_{1} g_{0}$ & $\mathrm{w}_{2} g_{0}$ \\
\hline $\mathrm{w}_{-2} g_{1}$ & $\mathrm{w}_{-1} g_{1}$ & $\mathrm{w}_{0} g_{1}$ & $\mathrm{w}_{1} g_{1}$ & $\mathrm{w}_{2} g_{1}$ \\
\hline $\mathrm{w}_{-2} g_{2}$ & $\mathrm{w}_{-1} g_{2}$ & $\mathrm{w}_{0} g_{2}$ & $\mathrm{w}_{1} g_{2}$ & $\mathrm{w}_{2} g_{2}$ \\
\hline
\end{tabular}

Fig. 3. Fractional derivative masks of the size $5 \times 5$ pixels ( $N=2$ ) obtained by multiplication and weighting the central row/column.

The weights matrix is further normalized: 


$$
\mathbf{W}_{\mathbf{N}}=\frac{1}{\sum_{k=-N}^{N} w_{k}}\left[w_{-N} \cdots w_{0} \cdots w_{N}\right]
$$

where:

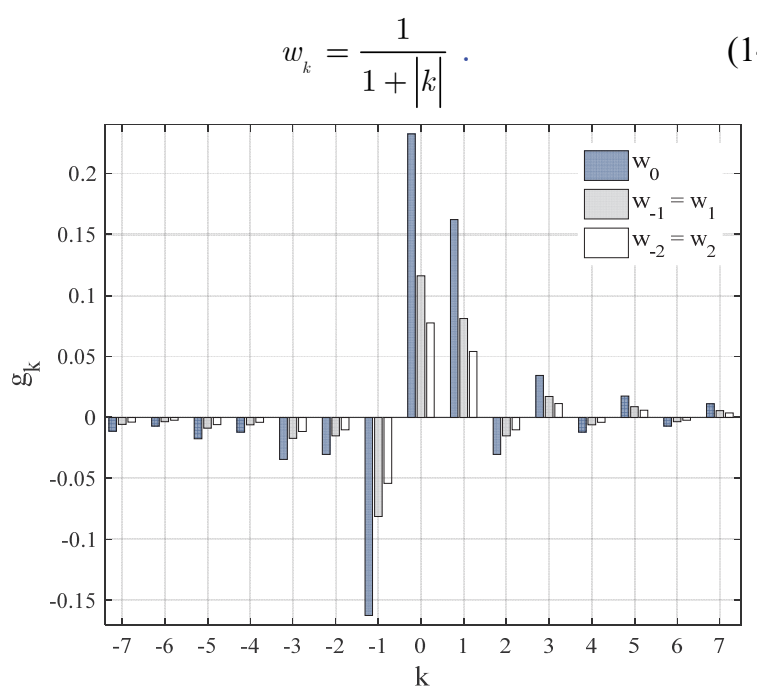

Fig. 4. Coefficients of the mask obtained using weighting of coefficients $g_{k}$ with three central weights: $\mathrm{w}_{0}, \mathrm{w}_{-1}=\mathrm{w}_{1}$ and $\mathrm{w}_{-2}=\mathrm{w}_{2}$. The order of differentiation in all three cases is

$$
\alpha=0.3 \text {. }
$$

Equation (14) shows that coefficients of the weight matrix, $\mathbf{W}_{\mathbf{N}}=\left[\mathrm{w}_{1, \mathrm{k}}\right]$, are symmetric, that is, $\mathrm{w}_{1,-k}=\mathrm{w}_{1, \mathrm{k}}$.

Weights masks are presented in Fig. 3. Also, weights matrix defined in this way adopts the reasoning of the Sobel operator where the central row ( $x$-directional mask) or column ( $y$-directional mask) is favored and the other rows/columns are attenuated with respect to vicinity to the central row/column. The coefficients of these masks are given in Fig. 4 for three coefficients from the weights matrix: $\mathrm{w}_{0}, \mathrm{w}_{-1}=\mathrm{w}_{1}$ and $\mathrm{w}_{-2}=\mathrm{w}_{2}$. Coefficients of the masks in this implementation are lower in intensity and the decrease with respect to vicinity to the central pixel is stronger than in the direct implementation.

\section{GRADIENT MASKs Evaluation}

\section{A. Experiment setup}

Images used for the assessment of previously analyzed fractional gradient masks are the first 10 chest radiographs (JPCLN001: JPCLN010) from the SCR database [8]. Radiographs are of the size $2048 \times 2048$ pixels with 16 bit depth.

For numerical evaluation of the analyzed masks we used signal-to-noise ratio (SNR) of the image defined as:

$$
S N R(I)=10 \log _{10} \frac{\mu_{I}}{\sigma_{I}}
$$

where $\mu_{I}$ denotes a mean value and $\sigma_{I}$ denotes a standard deviation of the image $I$.

Effective average gradient (EAG) is used to quantify the preservation of the edge-like content in the image [9]:

$$
E A G(I)=\frac{T G(I)}{T P(I)}
$$

where $T G$ is the total sum of the (first-order) gradient pixels, while $T P$ stands for the total number of pixels with non-zero (first-order) gradient.

Magnitude of the gradient of the image is calculated as a norm over gradients calculated for $x$ and $y$ direction. In case of Euclidean norm, that is most often used when operating with gradients [10], it is calculated as:

$$
M_{e}=\sqrt{G_{x}^{2}+G_{y}^{2}} .
$$

When applying infimum norm, magnitude is calculated as:

$$
M_{i}=\min \left\{\left|G_{x}\right|,\left|G_{y}\right|\right\}
$$

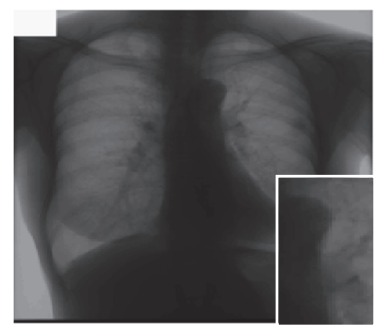

a)

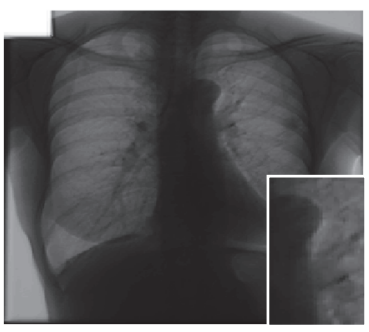

b)

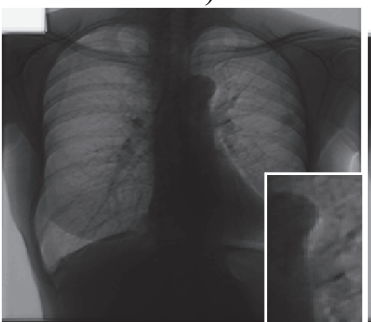

d)

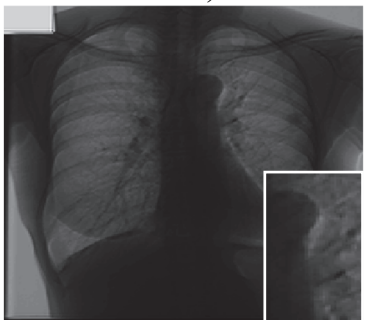

f)

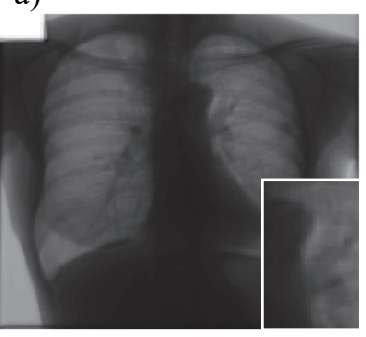

c)

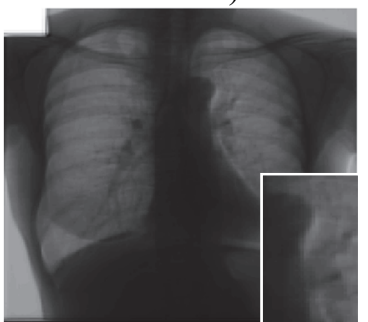

e)

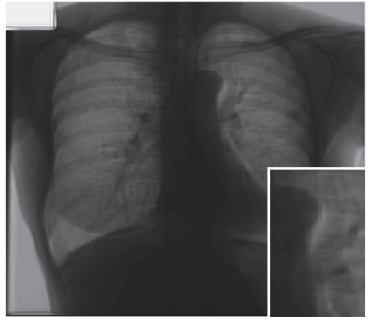

g)
Fig. 5. Illustration of the enhancement of a radiographic image using fractional derivative schemes and Euclidean norm: a) Original radiograph; b) direct implementation mask of order 0.3 ; c) weighting implementation mask of order 0.3 ; d) direct implementation mask of order 0.5 ; e) weighting implementation mask of order 0.5 ; f) direct implementation mask of order $0.7 ; \mathrm{g}$ ) weighting implementation mask of order 0.7 . All masks are of the same size of $7 \times 7$ pixels $(N=3)$. 


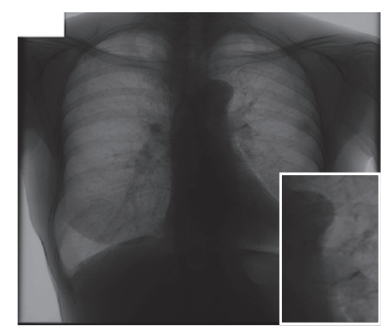

a)

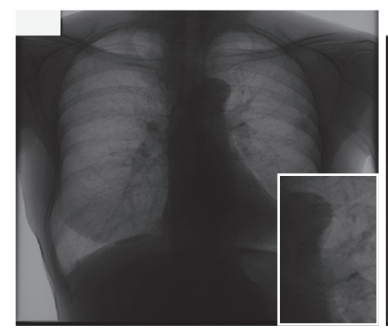

c)

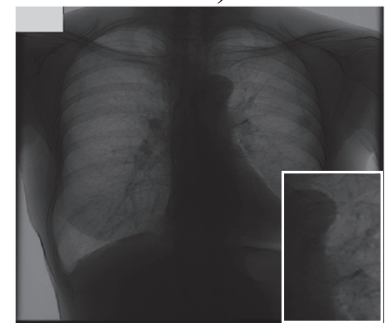

e)

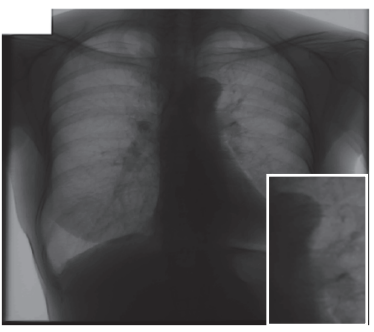

b)

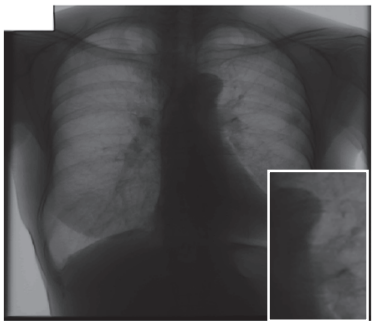

d)

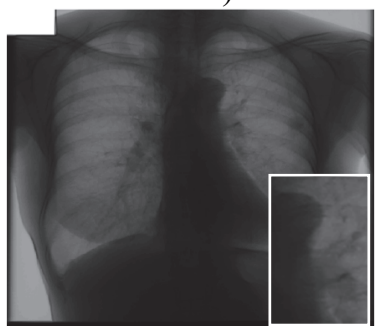

f)
Fig. 6. Illustration of the enhancement of a radiographic image using fractional derivative schemes and infimum norm:

a) direct implementation mask of order 0.3 ; b) weighting implementation mask of order 0.3 ; c) direct implementation mask of order 0.5 ; d) weighting implementation mask of order 0.5 ; e) direct implementation mask of order 0.7 ; ) weighting implementation mask of order 0.7. All masks are of the same size of $7 \times 7$ pixels $(N=3)$, while the original image is shown in Fig. 5. a).

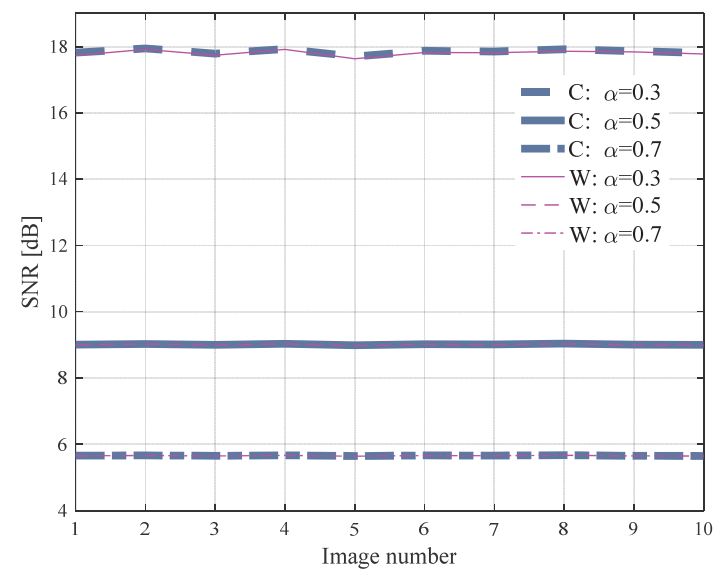

Fig. 7. SNR values calculated for direct (C) and weighting (W) implementation of the differentiation mask. Euclidean norm is applied. Order of differentiation, $\alpha$, is varying.

\section{B. Experimental evaluation}

Two parameters of the fractional gradient masks have a major influence on the radiographic image enhancement. In the first place it is the order of differentiation. Since the orders of differentiation closer to zero give images almost unchanged, while the orders closer to one provide more (first) gradient-like images, it is expected that fractional gradients of orders between zero and one will enhance the contrast of the image. This is more evident from Fig. 5 where both mask implementations are used for three orders of differentiation. Here we calculated gradient magnitudes using the Euclidean norm. Results obtained in the same test but using infimum norm are shown in Fig. 6. Quantitatively this is further confirmed with SNR (Fig.7 and Fig.8.) and EAG (Fig. 9. and Fig.10.) values. SNR values suggest that both implementations, direct and using a weight matrix, provide almost the same image quality, while the lower orders of differentiation give higher SNR values. When comparing the used two norms, it is evident that SNR values favor the Euclidean norm. On the other hand, infimum norm produces magnitude images with less evident artifacts around edges. EAG values favor direct implementation as it is expected from Fig.9 and Fig.10, since the textural content (details) is enhanced. It is also noticeable that higher orders of differentiation diminish EAG irrespective of the used metric. In case of infimum norm two implementations of gradient mask coefficients produce less noticeable differences in performance with respect to EAG values. The Euclidean norm, on the other hand, makes a clear distinction between those implementations. Further, EAG performance is better in case of the Euclidean norm, when using a direct implementation, while infimum norm gives better EAG results when using a weight matrix.

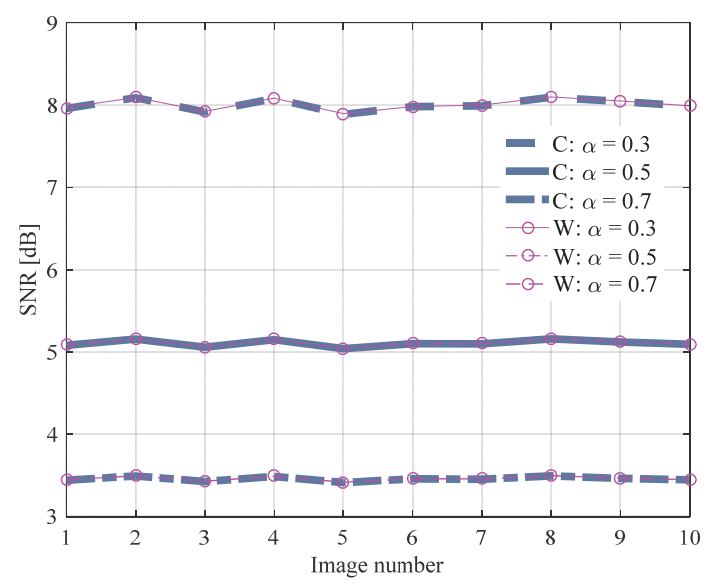

Fig. 8. SNR values calculated for direct (C) and weighting (W) implementation of the differentiation mask. Infimum norm is applied. Order of differentiation, $\alpha$, is varying.

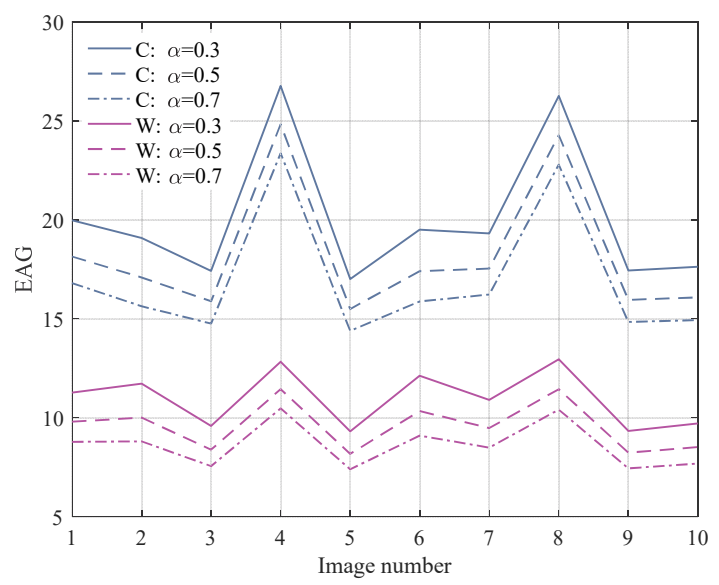

Fig. 9. EAG values calculated for direct (C) and weighting (W) implementation of the differentiation mask. Euclidean norm is applied. Order of differentiation, $\alpha$, is varying. 


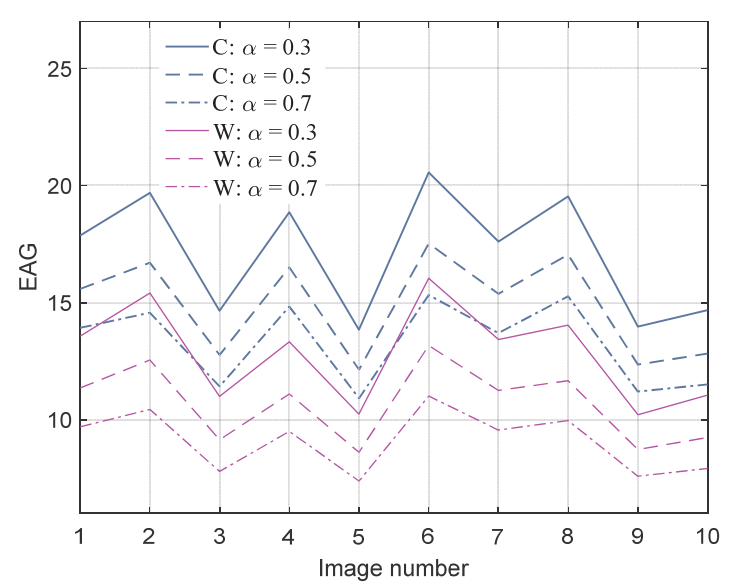

Fig. 10. EAG values calculated for direct (C) and weighting (W) implementation of the differentiation mask. Infimum norm is applied. Order of differentiation, $\alpha$, is varying.

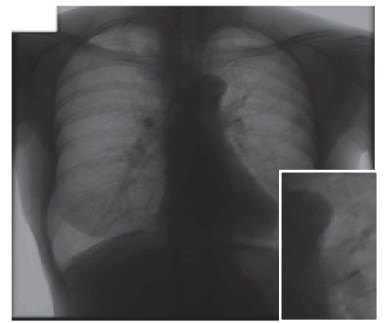

a)

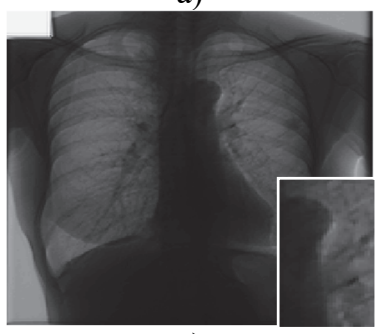

c)

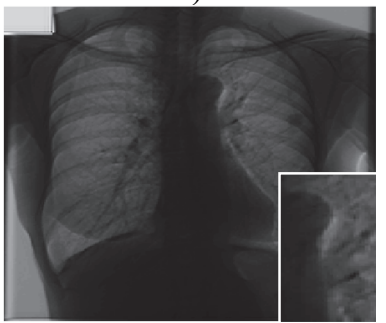

e)

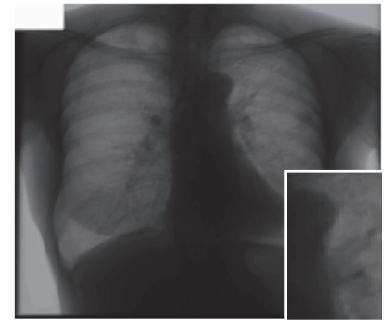

b)

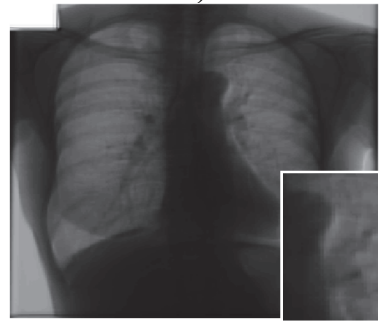

d)

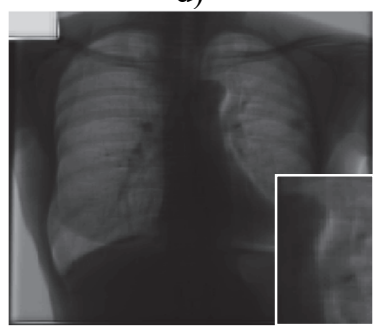

f)
Fig. 11. Influence of the mask sizes on fractional differentiation (Euclidean norm) of a radiographic image from Fig. 5. a): a), b) 3x3 mask; c), d) 7x7 mask; e), f) $11 \times 11$ mask. Images obtained using direct implementation masks are given on the left, while images obtained using weighting masks are given on the right side. The order of

differentiation is the same in all images and it is 0.5 .

The other parameter with impact on enhancement is the size of the gradient masks, $N$. Visual inspection of Fig.11 and Fig.12 suggests that direct implementation is more sensitive to mask size in case when the Euclidean norm is applied (Fig.11). Implementation of infimum norm produces images without artifacts on the edges (Fig.12). SNR values (Fig.13 and Fig.14) do not privilege, again, neither of implementations, while Euclidean norm gives better results with respect to SNR. EAG values (Fig.15 and Fig.16) give advantage to direct implementation. Also, EAG values are not significantly influenced by the mask size and implementation with weighting is more sensitive to mask size irrespective of applied norm. This is the consequence of both altering the coefficients of the weight matrix and inclusion of all the pixels within the mask (all coefficients of the mask are non-zero).

Gradient mask in weighting implementation includes all the pixels in the neighborhood and it produces averaging or smoothing of the image. It is especially visible on the edges in the images in Fig.5 and Fig. 12, in case of the Euclidean norm, and in Fig.6 and Fig.13, in case of infimum norm.

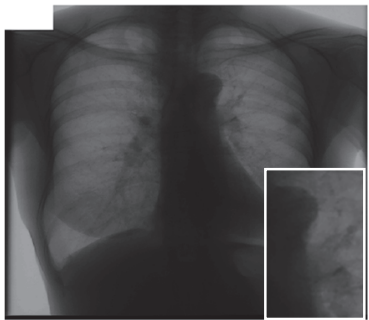

a)

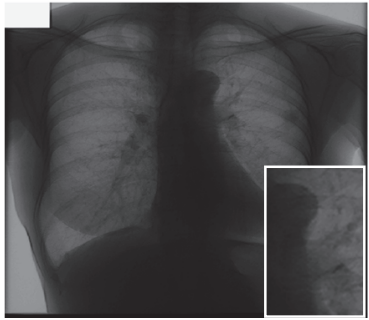

c)

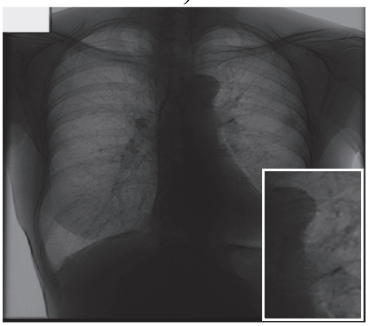

e)

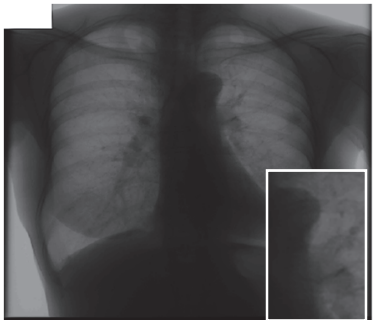

b)

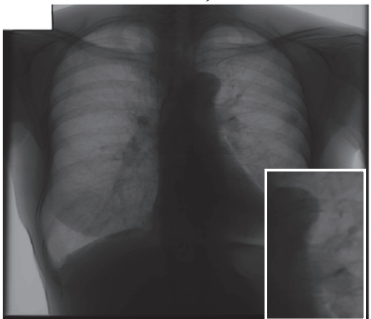

d)

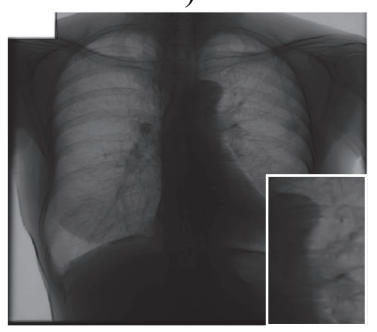

f)
Fig. 12. Influence of mask sizes on fractional

differentiation (Euclidean norm) of a radiographic image from Fig. 5. a): a), b) 3x3 mask; c), d) 7x7 mask; e), f) 11x11 mask. Images obtained using direct implementation masks are

given on the left, while images obtained using weighting masks are given on the right side. The order of differentiation is the same in all images and it is 0.5 .

\section{V.CONCLUSION}

Experiments conducted in this paper show that two analyzed realizations of $2 \mathrm{D}$ fractional derivative masks can be used for the enhancement of radiographic images. The first, that is, direct implementation results in images with enhanced details, while the second implementation or the implementation with a weight matrix possesses a smoothing effect and acts better in transition regions. When comparing norms used in this paper, numerical tests, considering SNR and EAG values, suggest the Euclidean norm. Visually undesirable effects on edges, however, are suppressed when using infimum norm.

Future research will include development of new fractional gradient operators based on central fractional differences and its application to radiographic images. 


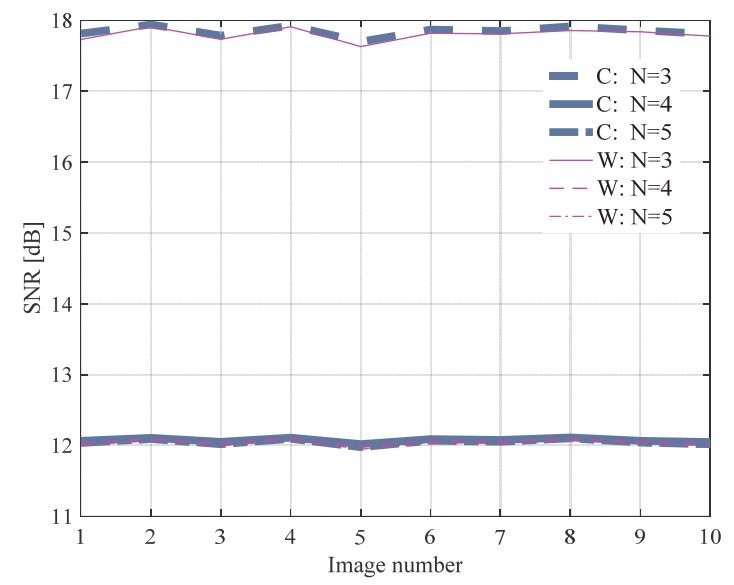

Fig. 13. SNR values calculated for direct (C) and weighting (W) implementation of the differentiation mask. Euclidean norm is applied. Mask size parameter, $N$, is varied.

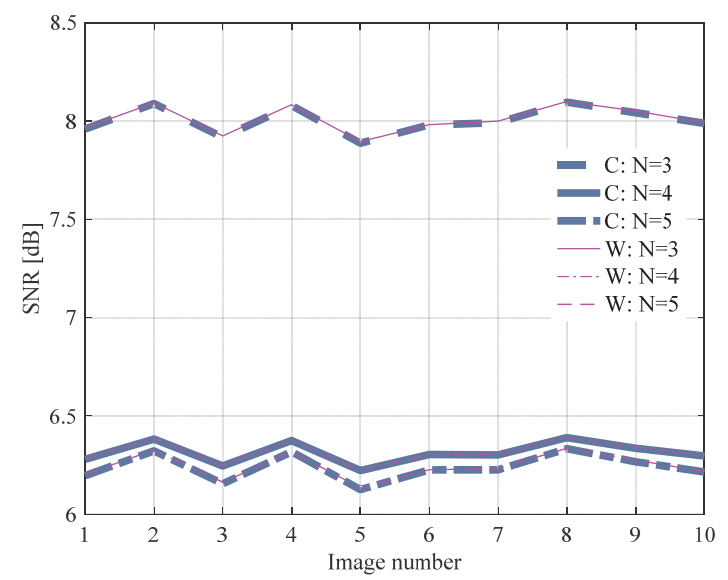

Fig. 14. SNR values calculated for direct $(\mathrm{C})$ and weighting (W) implementation of the differentiation mask. Infimum norm is applied. Mask size parameter, $N$, is varied.

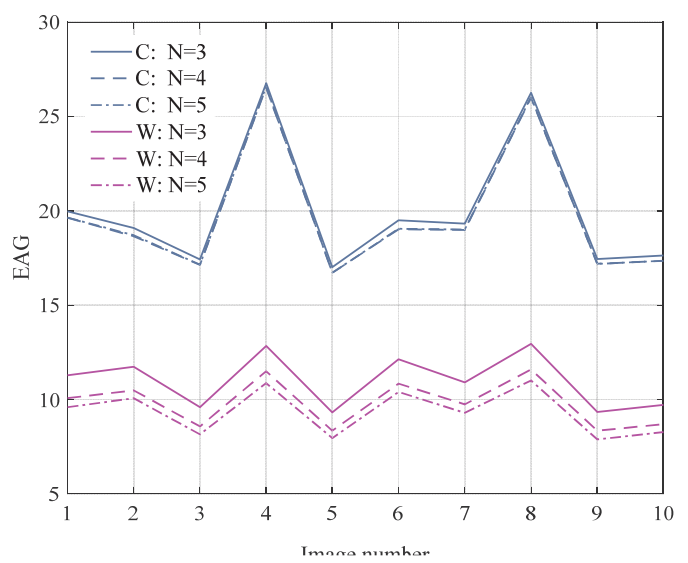

Fig. 15. EAG values calculated for direct (C) and weighting (W) implementation of the differentiation mask. Euclidean norm is applied. Mask size parameter, $N$, is varied.

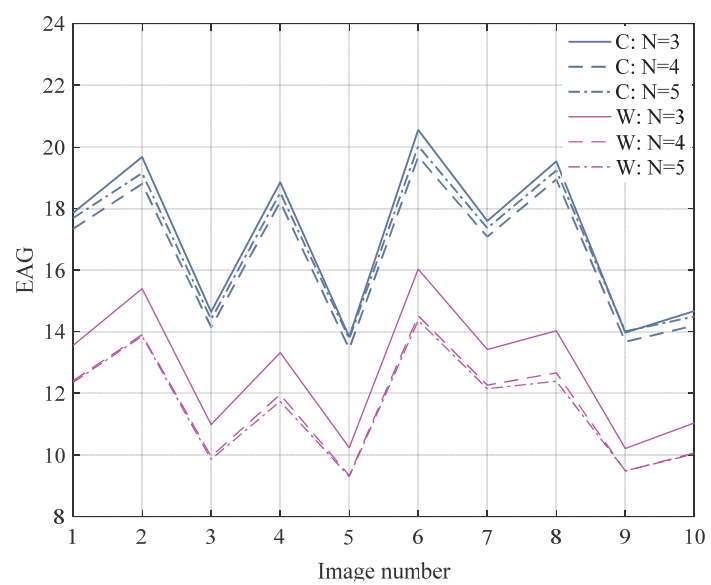

Fig. 16. EAG values calculated for direct $(\mathrm{C})$ and weighting (W) implementation of the differentiation mask. Infimum norm is applied. Mask size parameter, $N$, is varied.

\section{REFERENCES}

[1] I. Podlubny, Fractional Differential Equations: An Introduction to Fractional Derivatives, Fractional Differential Equations, to Methods of their Solution and some of their Applications, Mathematic. Academic Press, 1999.

[2] Yi-Fei Pu, Ji-Liu Zhou, and Xiao Yuan, "Fractional Differential Mask: A Fractional Differential-Based Approach for Multiscale Texture Enhancement," IEEE Trans. Image Process., vol. 19, no. 2, pp. 491-511, Feb. 2010

[3] J. Bai and X.-C. Feng, "Fractional-Order Anisotropic Diffusion for Image Denoising," IEEE Trans. Image Process., vol. 16, no. 10, pp. 2492-2502, Oct. 2007.

[4] M. D. Ortigueira and J.A.Tenreiro Machado, "What is a fractional derivative?," J. Comput. Phys., vol. 293, pp. 4-13, Jul. 2015.

[5] M. D. Ortigueira, Fractional Calculus for Scientists and Engineers, vol. 84. Springer Netherlands, 2011.

[6] M. P. Paskaš, I. S. Reljin, and B. D. Reljin, "Novel Fractional-Order Difference Schemes Reducible to Standard Integer-Order Formulas," IEEE Signal Process. Lett., vol. 24, no. 6, pp. 912-916, Jun. 2017.

[7] I. Sobel and G. Feldman, "A 3x3 Isotropic Gradient Operator for Image Processing," 1968.

[8] "SCR database, Image Sciences Institute." [Online]. Available: http://www.isi.uu.nl/Research/Databases/SCR/download.php. [Accessed: 03-Oct-2017].

[9] Y. J. Zhang and J. J. Gerbrands, "Transition region determination based thresholding," Pattern Recognit. Lett., vol. 12, no. 1, pp. 13-23, 1991.

[10] R. C. Gonzalez and R. E. Woods, Digital Image Processing, 3rd ed. Prentice-Hall, Inc., 2007.

[11]M. P. Paskaš, B. D. Reljin and I. S. Reljin, "Assessment of fractional derivative schemes for enhancement of chest radiographs," 201725 th Telecommunication Forum (TELFOR), Belgrade, 2017, pp. 1-4. 